\title{
Frequency and Causes of Delirium in Pediatric Intensive Care Unit: A Prospective Observational Study
}

\author{
Ahmet Yontem ${ }^{1} \oplus$, Dincer Yildizdas $²$, Ozden $\mathrm{O} \mathrm{Horoz}^{3} \oplus$, Faruk Ekinci ${ }^{4} \odot$, Merve Misirlioglu ${ }^{5} \odot$
}

\begin{abstract}
Background: The number of studies evaluating delirium and its frequency in critically ill infants, children, and adolescents is increasing day by day. The primary objective of this study was to evaluate all patients hospitalized in our pediatric intensive care unit (PICU) in terms of pediatric delirium, to determine the frequency and risk factors of pediatric delirium.

Patients and methods: The patients included in this study had been hospitalized in the PICU between November 1, 2018, and August 31, 2019, and were followed up for more than 48 hours.

Results: Delirium was detected in 14 patients (9.9\%) through regular evaluations. The Pediatric Index of Mortality 2 (PIM2) scores and the length of stay in the PICU were higher in patients with delirium ( $p=0.03$ and $p=0.01$, respectively). The use of respiratory support, sedationanalgesia, vasoactive and corticosteroid treatments, and physical restraints were higher in patients with delirium and were statistically significant $(p<0.05)$. Following admission to the PICU, psychosocial interventions were implemented for $76.1 \%$ of the whole cohort. Delirium developed in only five (4.5\%) of the 108 patients who underwent psychosocial interventions, while it was detected in nine (26.5\%) of the 34 patients who did not receive psychosocial interventions $(p=0.001)$. The psychosocial intervention was associated with a lower likelihood of delirium (odds ratio [OR], $0.237 ; p=0.044)$. An increasing number of days in the PICU was independently associated with increasing odds of delirium (OR, $1.095 ; p=0.037$ for each day).

Conclusions: We observed that the risk factors associated with delirium were similar to previous studies. Additionally, psychosocial intervention before delirium symptoms developed was associated with a lower risk of developing delirium. However, multicenter randomized controlled trials are needed on this subject.

Keywords: Delirium, Frequency, Prevention, Risk factors.

Indian Journal of Critical Care Medicine (2021): 10.5005/jp-journals-10071-23857
\end{abstract}

\section{INTRODUCTION}

Delirium is a neuropsychiatric syndrome that can be triggered by a variety of medical, surgical, pharmacological, and traumatic causes and can develop in critical patients of all ages. ${ }^{1,2}$ In the Diagnostic and Statistical Manual of Mental Disorders, five criteria are taken into consideration when defining delirium. Criteria $A$ and $C$ describe disturbances in attention and cognitive function. Criterion $B$ describes short-term baseline changes and a fluctuating course of symptoms. Criterion $D$ requires that delirium cannot be explained by another preexisting, established, or evolving neurocognitive disorder, or does not occur in the context of a severely reduced level of arousal. In addition, criterion $E$ requires evidence of findings that the disturbance is a consequence of another medical condition, substance intoxication or withdrawal, or exposure to a toxin, or that it is due to multiple etiologies. ${ }^{3}$

While high rates of the incidence of delirium have been reported in adult critical patients, the number of studies evaluating delirium and its frequency in critically ill infants, children, and adolescents is increasing day by day. ${ }^{4-8}$ The frequency of delirium in pediatric intensive care units (PICUs) is observed to be between 10 and $30 \%$, while the frequency of delirium in mechanically ventilated pediatric patients has been shown to reach up to $53 \%$. $^{4,5,9-11}$ Critical illness is one of the most common causes of delirium in children; ${ }^{1,2}$ however, delirium has been found to be associated with longer hospitalizations, higher treatment costs, and increased morbidity and mortality, even in children with lower severity of illness. ${ }^{12}$ Delirium can affect the social life of children after discharge as well as their clinical status during hospitalization. It has been reported
${ }^{1}$ Department of Pediatric Intensive Care, Cukurova University Medicine Faculty, Adana, Turkey

Corresponding Author: Ahmet Yontem, Department of Pediatric Intensive Care, Cukurova University Medicine Faculty, Adana, Turkey, Phone: +905075097709, e-mail: drayontem@gmail.com

How to cite this article: Yontem A, Yildizdas D, Horoz OO, et al. Frequency and Causes of Delirium in Pediatric Intensive Care Unit: A Prospective Observational Study. Indian J Crit Care Med 2021;25(6):715-719.

Source of support: Nil

Conflict of interest: None

that delirium may cause long-term cognitive impairment in survivors, and approximately one-third of children may experience post-traumatic stress disorder for the rest of their lives. ${ }^{13-15}$

The primary objective of this study was to evaluate the frequency and risk factors of pediatric delirium. The secondary objective of this study was to observe the association between psychosocial interventions and delirium prevalence in the PICU.

\section{Materials and Methods Study Design}

This single-center prospective study was conducted in an academic tertiary PICU that admitted patients with various illnesses between November 1, 2018, and August 31, 2019. The study was approved by the Institutional Review Board of Çukurova University Faculty of 
Medicine (2018/82). Written informed consent was obtained from the patients' relatives for their anonymized information.

Our PICU is an eight-bed multidisciplinary pediatric unit in a tertiary care university hospital. During the study period, 367 patients from all pediatric specialties, including various surgeries, were followed up. Excluded from the study were 81 patients with scores of five and six on the Pediatric Cerebral Performance Category (PCPC) scale and six patients with scores of -4 and -5 according to Richmond Agitation and Sedation Score (RASS) during their PICU stay, 36 patients who were comatose during their entire PICU stay, six patients who were already delirious at the time of admission, and 96 patients who were hospitalized in the PICU for less than 48 hours. The remaining 142 patients followed up in the PICU have been included in the study (Fig. 1).

Age, gender, height, weight, primary diagnosis, concomitant illness, Pediatric Index of Mortality 2 (PIM2) score, and the developmental status of the patients at the time of admission were recorded. The patients were evaluated in terms of their need for, and the length of, respiratory support, sedation-analgesia, muscle relaxants, and corticosteroid and vasoactive drug treatments during hospitalization. Antiepileptic use, sepsis, surgical procedures, hyperglycemia, use of physical restraints, and delirium onset time, length of delirium, and recurrent episodes of delirium in the patients diagnosed with delirium were recorded.

\section{Setting and Population}

Intensive care environmental modifications, strategies to promote sleep, day and night orientations, and communication with a child were specified based on the definitions in the previous study. ${ }^{16}$ All of the implementations are given in Table 1. The implementations mentioned in the standard of care were fulfilled for all patients. The parents were allowed to stay with the patients as long as the service conditions were appropriate. The additional implementations were intended to be fulfilled for all patients. The patients were considered as without psychosocial intervention in cases of (1) inability to communicate with the patient due to different mother tongue, (2) the patients for whom it was not appropriate for their parents to stay, and (3) none of the additional implementations could be fulfilled and it was not appropriate for the parents to stay with the patient.

\section{Measurement and Tools}

The patients were regularly evaluated by the nurses for sedation and pain conditions, and necessary pharmacological arrangements were made to avoid excessive sedation. The sedation status of the patients was recorded by the nurses at 4-hour intervals using the RASS. ${ }^{17}$ Delirious children with a RASS of 0 to -3 were considered to have hypoactive delirium and those with a score of 0 to +4 were considered to have hyperactive delirium. Delirious children with both negative and positive scores in the RASS scoring system were considered to have mixed delirium. Each child included in the study was evaluated for pediatric delirium using the Cornell assessment of pediatric delirium (CAPD) twice a day (at 08.00 and 16.00) by one of three trained fellow residents (A.Y., F.E., or M.M.) in accordance with our PICU standards. ${ }^{18}$ Each child with at least two consecutive CAPD scores $\geq 9$ was categorized as delirious. A study concerning the validity and reliability of CAPD scoring was previously conducted in Turkey. ${ }^{19}$ The PCPC scale was used to define any developmental delay in the patients. ${ }^{20}$ We used the PIM2 score to quantify the severity of illness at the point of admission. ${ }^{21}$ High-flow oxygen therapy, noninvasive ventilation,

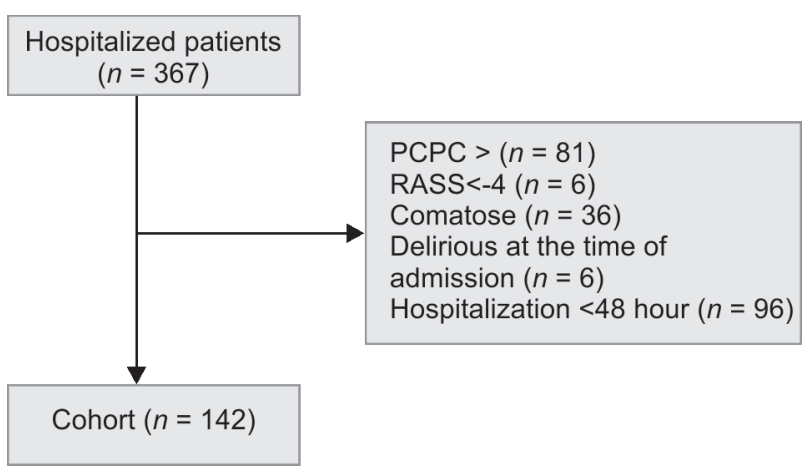

Fig. 1: Patients admitted to the pediatric intensive care unit

Table 1: Pediatric intensive care unit implementations for delirium Standard of care

- Dim the lighting in the PICU at night in order to contribute to the sleep cycle

- Wake the child at the same time each morning

- Place bed in a chairlike position when child tolerates

- Addressed by their names

- Explain painful interventions to the patients in a way that they could understand before performed

- Children who need glasses or hearing aids should wear them when possible

- Mute the redundant alarm sounds of medical devices

- Allow the parents to stay with the patients

- Oral treatments are administered by the parents

Additional implementations

- Have on hand favorite toys, blankets, music, and personal items

- Allow to spend time on phones or tablets

- Decorate room with familiar pictures of home, family, friends, and favorite pets

- Make the patients watch television in accordance with their age

and invasive ventilation supports were defined as respiratory support. Patients with height for age $<3 p$ were considered to have growth delay. ${ }^{22}$

Delirium onset time was defined as the number of days from admission to the PICU to the first diagnosis of delirium; length of delirium was defined as the number of days spent in delirium while hospitalized in the PICU. Recurrent delirium was defined as the second period of delirium, following at least 24 hours of normal mental status after recovery from the first period of delirium, during the same hospitalization.

\section{Statistics}

The description of the subjects is based on the entire cohort. Variables were summarized with counts and percentages, or the median and interquartile range (IQR). The one-way relationships between related variables and delirium status were analyzed by chi-square or Fisher's exact tests. Mann-Whitney $U$ tests were used to compare numerical measurements between groups. We used generalized linear models to estimate relative risk in univariate analyses of continuous variables and delirium. A value of $p<0.05$ was considered statistically significant. Stepwise multiple logistic 
regression analysis was performed by using variables that were found statistically significant. All statistical analyses were performed using SPSS 20 (IBM Corporation, Armonk, New York).

\section{Results}

The number of patients hospitalized in the PICU between November 1, 2018, and August 31, 2019, that were followed up for more than 48 hours and had a PCPC scale score of $<5$ was 142 . Of all the patients, 85 were boys (59.9\%) (Table 2). The median age was 43.0 months (IQR, 17-123). Postoperative care was the most common reason for admission, accounting for $39.4 \%$ of patients. The expected mortality rate, according to PIM2 scoring, was 4.0 (IQR, 1.4-11.0\%). The median length of stay in the PICU was 6 days (IQR, 4-9). Only one death occurred among patients without delirium, while no deaths occurred among delirious patients. The description of patients is shown in Table 2.

Delirium was detected in 14 patients (9.9\%) through regular evaluations. Eight patients (57.1\%) had hyperactive delirium, four (28.6\%) had mixed type delirium and two (14.3\%) had hypoactive delirium. While the median delirium development day was 4.5 days, at the earliest, delirium developed on the third day, and at the latest, it developed on the 29th day. Pharmacological treatment was not applied to any patient with delirium. The median length of delirium was 1.5 days. The second episode of delirium did not develop in any patients.

The characteristics of the patients with and without delirium were compared. No difference was found between the two groups in terms of age and gender ( $p=0.69$ and $p=0.43$, respectively), while the PIM2 score and length of PICU stay were higher in patients with delirium ( $p=0.03$ and $p=0.01$, respectively). The presence of delirium was associated with the use of respiratory support, sedation-analgesia, vasoactive and corticosteroid treatments, and physical restraints in univariate analysis $(p<0.05)$. However, the presence of delirium was not associated with the length of these treatments, except for vasoactives and corticosteroids. Growth and developmental delay, antibiotic use, and surgical procedures did not have any effect on delirium development. The relationship between delirium and the applications and treatments administered to patients is shown in Table 3.

Table 2: Description of subjects

\begin{tabular}{lc}
\hline & All patients $(n=142)$ \\
\hline Age (months) & $43.0(17-123)$ \\
Male & $85(59.9)$ \\
Reason for PICU admission & \\
Surgical procedure & $56(39.4)$ \\
Respiratory insufficiency & $21(14.8)$ \\
Neurologic disease & $12(8.5)$ \\
Renal/metabolic disorder & $10(7.0)$ \\
Trauma & $9(6.3)$ \\
Sepsis & $9(6.3)$ \\
Growth delay & $59(41.5)$ \\
Developmental delay & $38(26.8)$ \\
Psychosocial intervention & $108(76.1)$ \\
PIM2 & $4.0(1.4-11.0)$ \\
Death & $1(0.7)$ \\
Length of PICU stay (days) & $6(4-9)$ \\
\hline
\end{tabular}

PICU: Pediatric intensive care unit, PIM: Pediatric Index of Mortality 2 score
Table 3: The relationship between delirium and the characteristics, applications, and treatments administered to patients

\begin{tabular}{|c|c|c|c|}
\hline & \multicolumn{2}{|l|}{ Delirium } & \multirow[b]{2}{*}{$p$ value } \\
\hline & $\begin{array}{l}\text { No } \\
(n=128)\end{array}$ & $\begin{array}{l}\text { Yes } \\
(n=14)\end{array}$ & \\
\hline Age (months) & $42.0(16-127)$ & $51(22-123)$ & 0.69 \\
\hline Male & $78(60.9)$ & $7(50)$ & 0.43 \\
\hline Psychosocial intervention & $103(80.5)$ & $5(35.7)$ & 0.001 \\
\hline Respiratory support & $56(43.8)$ & $10(71.4)$ & 0.04 \\
\hline $\begin{array}{l}\text { Length of respiratory } \\
\text { support (days) }\end{array}$ & $4(1.0-8.5)$ & $4.5(1.8-15.0)$ & 0.57 \\
\hline Narcotic & $23(18.0)$ & $6(42.9)$ & 0.03 \\
\hline Length of narcotic (days) & $2(1.0-4.0)$ & $2.5(1.8-7.0)$ & 0.34 \\
\hline Benzodiazepine & $32(25.0)$ & $7(50.0)$ & 0.04 \\
\hline $\begin{array}{l}\text { Length of benzodiazepine } \\
\text { (days) }\end{array}$ & $2(1.0-3.8)$ & $3.0(2.0-5.0)$ & 0.16 \\
\hline Muscle relaxant & $9(7.0)$ & $3(21.4)$ & 0.06 \\
\hline $\begin{array}{l}\text { Length of muscle relaxant } \\
\text { (days) }\end{array}$ & $3(2.5-3.0)$ & $3(1-3)$ & 0.44 \\
\hline Vasoactive & $30(23.4)$ & $7(50.0)$ & 0.03 \\
\hline Vasoactive inotropic score & $5(5-12)$ & $10(7-25)$ & 0.06 \\
\hline $\begin{array}{l}\text { Length of vasoactive } \\
\text { (days) }\end{array}$ & $2(2-3)$ & $4.5(2.8-9.3)$ & 0.02 \\
\hline Corticosteroid & $45(35.2)$ & $9(64.3)$ & 0.03 \\
\hline $\begin{array}{l}\text { Length of corticosteroid } \\
\text { (days) }\end{array}$ & $3(2.0-4.3)$ & $5(3.5-6.5)$ & 0.01 \\
\hline Blood-product & $33(25.8)$ & $4(28.6)$ & 0.82 \\
\hline Antiepileptic & $20(15.6)$ & $4(28.6)$ & 0.22 \\
\hline Antibiotic & $54(42.2)$ & $6(42.9)$ & 0.96 \\
\hline Surgical procedure & $63(49.2)$ & $7(50.0)$ & 0.95 \\
\hline Physical restraint & $36(28.1)$ & $9(64.3)$ & 0.006 \\
\hline Growth retardation & $53(41.4)$ & $6(42.9)$ & 0.94 \\
\hline Developmental delay & $35(7.3)$ & $3(21.4)$ & 0.63 \\
\hline PIM2 & $3.4(1.2-9.4)$ & $23.7(2.4-43.4)$ & 0.03 \\
\hline Length of PICU stay (days) & $5(4-9)$ & $7.5(6-13)$ & 0.01 \\
\hline
\end{tabular}

PICU: Pediatric intensive care unit, PIM: Pediatric Index of Mortality 2 score

Following admission to the PICU, psychosocial interventions were implemented for $76.1 \%$ of the whole cohort of patients. Delirium developed in only five (4.5\%) of the 108 patients who underwent psychosocial interventions, while it was detected in nine (26.5\%) of the 34 patients who did not undergo psychosocial interventions ( $p=0.001)$. Using multivariable logistic regression adjusting for the admission PIM2 score, we found that presence of delirium was independently associated with psychosocial interventions and length of stay in the PICU (Table 4). The psychosocial intervention was associated with a lower likelihood of delirium (odds ratio [OR], 0.237; $p=0.044$ ). Increasing days in PICU were independently associated with increasing odds of delirium (OR, 1.095; $p=0.037$ for each day).

\section{Discussion}

Of the 142 patients screened, 14 patients developed delirium. Consistent with the current literature, the severity of illness, length of PICU stay, frequencies of respiratory support, narcotic, benzodiazepine, vasoactive and corticosteroid treatments, and 
Table 4: Multivariable logistic regression analyses predicting delirium $(n=142)$

\begin{tabular}{lll}
\hline & Adjusted odds ratio (95\% Cl) & p value \\
\hline Respiratory support & - & 0.502 \\
Narcotic & - & 0.512 \\
Benzodiazepine & - & 0.800 \\
Vasoactive & - & 0.354 \\
Corticosteroid & - & 0.246 \\
Physical restraint & - & 0.173 \\
PIM2 & - & 0.096 \\
Psychosocial intervention & & \\
Yes & $0.237(1.04-17.24)$ & 0.044 \\
No & $1.095(1.006-1.192)$ & 0.037 \\
Length of PICU stay & PICU: Pediatic intensivecar
\end{tabular}

PICU: Pediatric intensive care unit, PIM: Pediatric Index of Mortality 2 score

the use of physical restraints were higher in patients with delirium in our study. There were no differences between the patients with and without delirium in terms of gender, age, growth and developmental delay, blood-product support, antibiotic use, antiepileptic use, surgical procedures, and length of medical treatments (except corticosteroids). In our study, $76.1 \%$ of patients underwent psychosocial interventions as mentioned above. The frequency of delirium in the patients without psychosocial interventions was higher than in the patients with psychosocial interventions. Using multivariable logistic regression adjusting for the admission PIM2 score, we found that psychosocial interventions can reduce the likelihood of delirium 4.22 times. Nevertheless, the length of PICU stay can increase the likelihood of delirium 1.095 times for each day.

While delirium can develop in many circumstances during hospitalization, it is more frequently observed in PICUs due to the worsening of illnesses and increased exposure to pharmacological agents. The severity of illness, differences in patient population, sedation practices, and clinical norms may explain the difference in delirium frequency among institutions. In our study, the frequency of delirium was $9.9 \%$, slightly less than the literature data. We think that the general frequency of delirium was lower in our PICU because $76.1 \%$ of the patients underwent psychosocial interventions after being admitted.

Various studies have suggested various risk factors for the development of delirium; however, it has been found that, in general, $<5$ years of age, the severity of illness, the need for mechanical ventilation, vasopressors, benzodiazepines, narcotics, and the use of physical restraints are strongly associated with delirium, and delirium prevalence increases in direct proportion with a longer PICU stay. 4,23,24 The authors have reported that this may be related to the accumulation of modifiable iatrogenic risk factors throughout the course of the illness and unchangeable demographic characteristics. In the univariate analysis, the risk factors associated with delirium were generally consistent with the current literature. However, the presence of delirium was not associated with these risk factors in the multivariable model. We think that this association could have been established if more patients had been included in the study.

The number of studies evaluating the recognition, management, and even long-term outcomes of pediatric delirium has been increasing in recent years. Although delirium is known to cause an increase in mortality, morbidity, length of hospitalization, and treatment costs, ${ }^{25,26}$ there are not enough studies that focus on evaluating interventions to reduce the frequency of delirium. As always in health services, adopting the approach that "prevention is better than treatment" will reduce the risk of developing the illness. Simone et al. conducted a prospective study in a PICU for a period of 22 months, where delirium monitoring protocol was implemented. ${ }^{27}$ The frequency of delirium was observed to decrease from 19.3 to $11.8 \%$ after implementation of sedation and early mobilization protocols.

The workgroup of experts in the field of child and adolescent psychiatry established a flowchart for the monitoring and management of delirium in order to standardize patient care in children with delirium. This flowchart provides recommendations to clinicians under these headings: "Prevention and Identification of Pediatric Delirium" and "Diagnosis and Management of Pediatric Delirium." Recommendations for physiological, pharmacological, and environmental factors should be taken into account, according to the flowchart of "Prevention and Identification of Pediatric Delirium". ${ }^{28}$ One of the most important factors in delirium management, under these headings, is reducing the patient's anxiety by allowing the parents to stay with the patient. Where possible, factors such as allowing a parent or loved one to stay with the patient on a continuous basis, and having the same nurses caring for the patient, are beneficial for children with delirium. In our study, the frequency of delirium was $26.5 \%$ in patients without psychosocial interventions, while it was $4.5 \%$ in patients with psychosocial interventions $(p=0.01)$. We found that psychosocial interventions following admission to the PICU can reduce the likelihood of delirium 4.22 times.

Our study has some limitations. First, the study was conducted in a single academic PICU, which limits generalization to other institutions. Prior to the study, we did not have data on the prevalence of delirium in our PICU and we could not evaluate the exact effect of psychosocial interventions since it was not a randomized controlled trial. Another limitation was that the study was carried out during the day shift and children with symptoms of delirium at night were not taken into account. Therefore, the actual frequency of pediatric delirium in our clinic may be slightly higher than was measured. We presented the frequency of the delirium types based on a RASS score. However, further analysis was impossible due to the relatively small number of delirious children. Lastly, 36 patients who were at the high risk of delirium were excluded because of being comatose during their entire PICU stay. As a consequence, we observed low mortality.

In conclusion, we observed that the risk factors associated with delirium were similar to previous studies. Additionally, psychosocial intervention before delirium symptoms develop was associated with a lower risk of developing delirium. However, multicenter randomized controlled trials are needed on this subject.

\section{OrCID}

Ahmet Yöntem (1) https://orcid.org/0000-0002-9982-0310

Dincer Yildizdas @ https://orcid.org/0000-0003-0739-5108

Ozden O Horoz 나 https://orcid.org/0000-0001-7590-650X

Faruk Ekinci $\odot$ https://orcid.org/0000-0001-6675-3150

Merve Misirlioglu @ https://orcid.org/0000-0002-9554-841X 


\section{References}

1. Schieveld J, Ista E, Knoester H, Molag M. Pediatric delirium: a practical approach. In: Rey J, editor. IACAPAP e-textbook of child and adolescent mental health. Geneva, Switzerland: International Association for Child and Adolescent Psychiatry and Allied Professions; 2015 [chap 1.5:1-17]. Available from: http://iacapap.org/wp-content/uploads/I.5DELIRIUM-2015.pdf [Accessed February 16, 2017].

2. Traube C, Silver G, Gerber LM, Kaur S, Mauer EA, Kerson A, et al. Delirium and mortality in critically ill children: epidemiology and outcomes of pediatric delirium. Crit Care Med 2017;45(5):891-898. DOI: 10.1097/CCM.0000000000002324.

3. American Psychiatric Association. Diagnostic and statistical manual of mental disorders. 5th ed. American Psychiatric Association; 2013.

4. Silver G, Traube C, Gerber LM, Sun X, Kearney J, Patel A, et al. Pediatric delirium and associated risk factors: a single-center prospective observational study. Pediatr Crit Care Med 2015;16(4):303-309. DOI: 10.1097/PCC.0000000000000356.

5. Smith HA, Brink E, Fuchs DC, Ely EW, Pandharipande PP. Pediatric delirium: monitoring and management in the pediatric intensive care unit. Pediatr Clin North Am 2013;60(3):741-760. DOI: 10.1016/ j.pcl.2013.02.010.

6. Silver GH, Kearney JA, Kutko MC, Bartell AS. Infant delirium in pediatric critical care settings. Am J Psychiatry 2010;167(10):1172-1177. DOI: 10.1176/appi.ajp.2010.09111606.

7. Turkel SB, Jacobson J, Munzig E, Tavaré CJ. Atypical antipsychotic medications to control symptoms of delirium in children and adolescents. J Child Adolesc Psychopharmacol 2012;22(2):126-130. DOI: 10.1089/cap.2011.0084.

8. Turkel SB, Jacobson JR, Tavaré CJ. The diagnosis and management of delirium in infancy. J Child Adolesc Psychopharmacol 2013;23(5):352356. DOI: 10.1089/cap.2013.0001.

9. Silver G, Traube C, Kearney J, Kelly D, Yoon MJ, Nash Moyal W, et al. Detecting pediatric delirium: development of a rapid observational assessment tool. Intens Care Med 2012;38(6):1025-1031. DOI: 10.1007/ s00134-012-2518-z.

10. Smith HA, Boyd J, Fuchs DC, Melvin K, Berry P, Shintani A, et al. Diagnosing delirium in critically ill children: validity and reliability of the pediatric confusion assessment method for the intensive care unit. Crit Care Med 2011;39(1):150-157. DOI: 10.1097/ CCM.0b013e3181feb489.

11. Daoud A, Duff JP, Joffe AR, Alberta Sepsis Network. Diagnostic accuracy of delirium diagnosis in pediatric intensive care: a systematic review. Crit Care 2014;18(5):489. DOI: 10.1186/s13054-014-0489-x.

12. Smith HA, Berutti T, Brink E, Strohler B, Fuchs DC, Ely EW, et al. Pediatric critical care perceptions on analgesia, sedation, and delirium. Semin Respir Crit Care Med 2013;34(2):244-261. DOI: 10.1055/s-00331342987.

13. Girard TD, Jackson JC, Pandharipande PP, Pun BT, Thompson $J \mathrm{~L}$, Shintani AK, et al. Delirium as a predictor of long-term cognitive impairment in survivors of critical illness. Crit Care Med 2010;38(7):1513-20. DOI: 10.1097/CCM.0b013e3181e47be1.

14. MacLullich AMJ, Beaglehole A, Hall RJ, Meagher DJ. Delirium and long-term cognitive impairment. Int Rev Psychiatry Abingdon Engl 2009;21(1):30-42. DOI: 10.1080/09540260802675031.
15. Colville G, Kerry S, Pierce C. Children's factual and delusional memories of intensive care. Am J Respir Crit Care Med 2008;177(9):976-982. DOI: 10.1164/rccm.200706-8570C.

16. Bettencourt A, Mullen JE. Delirium in children: Identification, prevention, and management. Crit Care Nurse 2017;37(3):9-18. DOI: 10.4037/ccn2017692.

17. Kerson AG, DeMaria R, Mauer E, Joyce C, Gerber LM, Greenwald BM, et al. Validity of the Richmond Agitation-Sedation Scale (RASS) in critically ill children. J Intensive Care 2016;4:65. DOI: 10.1186/s40560016-0189-5.

18. Traube C, Silver G, Kearney J, Patel A, Atkinson TM, Yoon MJ, et al. Cornell Assessment of Pediatric Delirium: a valid, rapid, observational tool for screening delirium in the PICU. Crit Care Med 2014;42(3):656663. DOI: $10.1097 / C C M .0 b 013 e 3182 a 66 b 76$.

19. Ergin B, Arı M, Bayram B, Karapınarlı N, Kahraman C, Karakaya S, et al. Cornell pediatrik deliryum değerlendirilme ölçeğinin Türkçe'ye uyarlanması: Geçerlilik ve güvenilirlik çalışması. XV. Çocuk Acil Tıp ve Yoğun Bakım Kongresi 2018;144-147.

20. Fiser DH, Long N, Roberson PK, Hefley G, Zolten K, Brodie-Fowler $M$. Relationship of pediatric overall performance category and pediatric cerebral performance category scores at pediatric intensive care unit discharge with outcome measures collected at hospital discharge and 1- and 6-month follow-up assessments. Crit Care Med 2000;28(7):2616-2620. DOI: 10.1097/00003246200007000-00072.

21. Slater A, Shann F, Pearson G; Paediatric Index of Mortality (PIM) study group: PIM2: a revised version of the Paediatric Index of Mortality. Intensive Care Med 2003;29(2):278-285. DOI: 10.1007/s00134-0021601-2.

22. Neyzi O, Bundak R, Gökçay G, Günöz H, Furman A, Darendeliler F, et al. Reference values for weight, height, head circumference, and body mass Index in Turkish children. J Clin Res Pediatr Endocrinol 2015;7(4):280-293. DOI: 10.4274/jcrpe.2183.

23. Traube C, Silver G, Reeder RW, Doyle H, Hegel E, Wolfe HA, et al. Pediatric delirium in critically-ill children: an international point prevalence study. Crit Care Med 2017;45(4):584-590. DOI: 10.1097/ CCM. 0000000000002250 .

24. Hatherill S, flisher AJ. Delirium in children and adolescents: a systematic review of the literature. J Psychosom Res 2010;68(4):337344. DOI: 10.1016/j.jpsychores.2009.10.011.

25. Traube C, Mauer EA, Gerber LM, Kaur S, Joyce C, Kerson A, et al. Cost associated with pediatric delirium in the ICU. Crit Care Med 2016;44(12):1175-1179. DOI: 10.1097/CCM.0000000000002004.

26. Turkel SB, Tavare' CJ. Delirium in children and adolescents. J Neuropsychiatry Clin Neurosci 2003;15(4):431-435. DOI: 10.1176/ jnp.15.4.431.

27. Simone S, Edwards S, Lardieri A, Walker LK, Graciano AL, Kishk OA, et al. Implementation of an ICU bundle: an interprofessional quality improvement project to enhance delirium management and monitor delirium prevalence in a single PICU. Pediatr Crit Care Med 2017;18(6):531-540. DOI: 10.1097/PCC.0000000000001127.

28. Silver GH, Kearney JA, Bora S, De Souza C, Giles L, Hrycko S, et al. A clinical pathway to standardize care of children with delirium in pediatric inpatient settings. Hosp Pediatr 2019;9(11):909-916. DOI: 10.1542/hpeds.2019-0115. 